\title{
A SIMPLE MINIMAX ESTIMATOR FOR QUANTUM STATES
}

\author{
HUI KHOON NG \\ Centre for Quantum Technologies, National University of Singapore \\ 3 Science Drive 2, Singapore 117543, Singapore \\ and Applied Physics Lab, DSO National Laboratories \\ 20 Science Park Drive, Singapore 118230 \\ cqtnhk@nus.edu.sg \\ BERTHOLD-GEORG ENGLERT \\ Centre for Quantum Technologies, National University of Singapore \\ 3 Science Drive 2, Singapore 117543, Singapore \\ and Department of Physics, National University of Singapore \\ 2 Science Drive 3, Singapore 117542, Singapore \\ cqtebg@nus.edu.sg
}

Received 16 March 2012

\begin{abstract}
Quantum tomography requires repeated measurements of many copies of the physical system, all prepared by a source in the unknown state. In the limit of very many copies measured, the often-used maximum-likelihood (ML) method for converting the gathered data into an estimate of the state works very well. For smaller data sets, however, it often suffers from problems of rank deficiency in the estimated state. For many systems of relevance for quantum information processing, the preparation of a very large number of copies of the same quantum state is still a technological challenge, which motivates us to look for estimation strategies that perform well even when there is not much data. After reviewing the concept of minimax state estimation, we use minimax ideas to construct a simple estimator for quantum states. We demonstrate that, for the case of tomography of a single qubit, our estimator significantly outperforms the ML estimator for small number of copies of the state measured. Our estimator is always full-rank, and furthermore, has a natural dependence on the number of copies measured, which is missing in the ML estimator.
\end{abstract}

Keywords: Quantum tomography; state estimation; minimax; maximum likelihood; Bayesian

\section{Introduction}

Tomography is the art of estimating the state of a system put out by a given source. For example, one might be interested in characterizing the polarization of a photon from a laser source; or two parties in a communication protocol want to know the state they jointly receive from a common source; or an experimentalist might want to verify that a source built in his lab to provide some target state is indeed meeting its specifications. The scope of tomography can be broadened to include parameter estimation, that is, to estimate a certain quantity of interest for some 
operational task (the fidelity between output state and target state, for example, or the expectation value of some fixed observable on the state). In this work, however, we will only deal with the most often discussed case of estimating the full state.

Tomography involves two steps: (i) the measurement of identical copies of the state; (ii) the conversion of the data collected from the measurement into an estimator for the state. In the simplest case, the measurement step (i) involves the same measurement on every copy of the state. More generally, it can be adaptive, that is, the measurement to be made on the $k$ th copy can depend on information gathered from measuring the previous $k-1$ copies. In the estimation step (ii), the simplest method gives a point estimator, which is a single state that represents our best guess of the identity of the true state. More generally, one can give a set of states compatible with the observed data that includes the true state with high probability. Such region estimators are known in classical estimation theory, and have appeared recently in the quantum arena 122 In our work, we will discuss the simplest case of repeated (non-adaptive) measurements, particularly measurements with the property of being symmetric and informationally complete, and focus on the issue of providing a point estimator.

The most popular procedure leading to a point estimator goes under the collective name of maximum-likelihood (ML) methods, first applied to quantum tomography by Hradil ${ }^{3}$ ML methods prescribe as the point estimator the state with the largest likelihood of giving rise to the observed data, and there are numerous variations to this theme depending on the scenario in question (see Ref. 4 for a good review). ML methods are particularly attractive because they do not require the choice of a prior distribution, a problem that plagues alternative methods based on Bayesian ideas (see, for example, Refs. 5 and 6 ). In the limit of a very large number of copies of the state measured, ML methods work very well - the likelihood function becomes so sharply peaked around the true state that one requires little sophistication to make a good guess.

However, for small sample sizes, ML methods are perhaps less well-motivated, and there is reason, as we will see in Section 2.3. to look for other methods in the estimation step. Besides, one should hardly expect that ML methods are the best choice for all scenarios, since its optimality is based on a particular figure-of-merit@ and whether this is a suitable figure-of-merit will undoubtedly depend on the task at hand. This motivates us to look beyond ML methods for alternative strategies appropriate for different tasks.

${ }^{a}$ Here is a comment that will likely make sense to the reader only upon reading the remainder of the paper: The ML estimator can be shown to be optimal in terms of minimizing the average risk, where the averaging uses the prior distribution $\mathrm{d} \mu=\mathrm{d} p_{1} \mathrm{~d} p_{2} \ldots \mathrm{d} p_{K}$ for a state characterized by probabilities $\left\{p_{k}\right\}_{k=1}^{K}$. The estimation error is quantified by a cost function that assigns a value 0 only when the estimator and the true state are identical, and 1 otherwise (see, for example, Ref. 7). Hence, even though the ML estimator requires no choice of prior distribution in its construction, in judging its efficacy, one still requires a choice of prior distribution to quantify its average performance over all true states. This point will be reiterated later in the text. 
A class of estimation procedures that requires no arbitrary or subjective choice of prior distribution is the class of minimax methods. In a minimax procedure, one looks for an estimator, usually from within a specified class of estimators, that gives the smallest worst-case (over all true states) estimation error. This gives an optimality condition that holds regardless of the probability of occurrence of each true state. While such a "worst-case scenario" approach may be overly cautious for some purposes, it can be suitable and, in fact, necessary for tasks like cryptography where one would prefer to acknowledge ignorance rather than make a wrong guess.

Minimax procedures are, unfortunately, notoriously difficult to implement, even for classical problems. This is hardly surprising since they involve a double optimization - first a maximization of the estimation error over all possible true states, followed by a minimization of this maximum over the class of estimators under consideration.

However, if we employ the commonly used mean squared error to quantify the estimation error, a minimax estimator with particularly nice features is known for the problem of a $K$-sided classical die. While the minimax estimator for the quantum analog of this problem is not known, we demonstrate here a general procedure to obtain a point estimator for the quantum problem that retains most of the desirable features of the classical minimax solution. This estimator is not minimax in the set of all estimators, as is the case for its classical analog, but is minimax within a smaller class of estimators with mathematical structure motivated by the solution for the classical die problem. This quantum generalization of the minimax point estimator, despite being rather ad-hoc in its construction, performs remarkably well in comparison to ML estimators for the qubit case investigated in detail. Furthermore, the estimator is easy to use as it requires no complicated numerical optimization. It can find utility as a good first guess for tomographic experiments, particularly if one only has access to a small number of copies of the state. Applying a similar procedure to adapt other known estimators for the classical problem to the quantum case might be equally fruitful.

Our goal here is partly to review the use of minimaxity as a means of choosing an estimation procedure. This is, of course, well known in the classical estimation theory community. In the quantum context, however, while minimax ideas have appeared in the quantum state estimation literature (see, for example, Refs. 8.9 .10 ), they remain little explored. Here, we organize the ideas into a consistent programme (Section 2), and contribute by presenting a simple estimator for quantum states motivated by minimax considerations (Section 3). The geometrical properties of symmetric quantum measurements are discussed in an appendix, and two more appendices contain mathematical details.

\section{Minimax estimation}

We first review two types of estimators - maximum-likelihood and mean estimators - before leading up to the idea of minimaxity. We also review the well- 
studied problem of the classical die, which serves two purposes: first, to define the notation and provide a concrete example for the application of the different estimation procedures; second, to provide guidance in the quantum problem studied in the next section. The reader is to note, however, that the ideas of state estimation discussed in this section are equally applicable to the quantum problem. In moving to the quantum arena, there are significant differences in the setup of the problem that complicate the application of the state estimation procedures, but the ideas behind each procedure remain unchanged. This is a reminder that the classical state estimation literature has much to teach us, even in the quantum context.

\subsection{The classical $K$-sided die}

Consider a $K$-sided die with faces labeled $k=1,2, \ldots, K$. The probability that face $k$ turns up when the die is tossed is denoted by $p_{k}$. Tosses of the die are described by the probability distribution $\left\{p_{k}\right\}_{k=1}^{K}$, with $p_{k} \geq 0$ and $\sum_{k} p_{k}=1$. Suppose we are given a die for which the probabilities are unknown, and we are allowed $N$ tosses of that die to attempt an estimate of the $p_{k}$ values.

Let us discuss the tomography of a $K$-sided die using language suitable for quantum state tomography. We write the state of a die with probability distribution $\left\{p_{k}\right\}_{k=1}^{K}$ as $\rho=\sum_{k=1}^{K}|k\rangle p_{k}\langle k|$, where ket $|k\rangle$ represents face $k$ turning up in a toss of the die. We can think of $\{|k\rangle\}$ as a basis for the state space with an inner product defined such that $\langle k \mid l\rangle=\delta_{k l}$. A single toss of the die is then, in this language, a measurement in the basis $\{|k\rangle\}_{k=1}^{K}$.

We can describe this measurement formally as a probability operator measurement (POM) with outcomes $\Pi_{1}, \Pi_{2}, \ldots, \Pi_{K}$. To define a POM, the operators $\Pi_{k}$ must be non-negative and normalized to unit sum,

$$
\Pi_{k} \geq 0 \quad \text { for all } k, \quad \text { with } \quad \sum_{k} \Pi_{k}=1 .
$$

The measurement can be thought of as comprising $K$ detectors, each corresponding to one of the POM outcomes $\Pi_{k}$. The probability that the $k$ th detector clicks, if we have the input state $\rho$, is given by Born's rule

$$
p_{k}=\operatorname{tr}\left\{\Pi_{k} \rho\right\} .
$$

For the case of the $K$-sided die, the POM corresponding to a single toss of the die can be described using the $\mathrm{POM}\left\{\Pi_{k} \equiv|k\rangle\langle k|\right\}$, and $p_{k}$ is simply the probability that the face $k$ turns up in a single toss of the die.

To improve the efficiency of the tomographic measurement, one often chooses a POM that is symmetric (S). The properties of S-POMs are the subject of Appendix A. Here, we are content with considering S-POMs that have rank-1 outcomes, in which case

$$
\operatorname{tr}\left\{\Pi_{k} \Pi_{l}\right\}=\frac{d^{2}}{K^{2}}\left[\delta_{k l}+\frac{K-d}{(K-1) d}\left(1-\delta_{k l}\right)\right] \text { for all } k, l,
$$


where the dependence on $k$ and $l$ is in the Kronecker deltas only. In particular, for $K=d$, this covers the case of the classical die, for which this S-POM is informationally complete (IC). One then speaks of a SIC-POM

Given a SIC-POM, every state of the system can be written as

$$
\rho=\sum_{k=1}^{K} p_{k} \Lambda_{k},
$$

where $p_{k}$ is computed via the Born's rule of 2 for the SIC-POM, and the $\Lambda_{k} \mathrm{~s}$ are hermitian, unit-trace operators with $\operatorname{tr}\left\{\Pi_{k} \Lambda_{l}\right\}=\delta_{k l}$ as their defining property. Specifically, we have

$$
\Lambda_{k} \equiv \frac{(K-1) K}{(d-1) d}\left[\Pi_{k}-\frac{K-d}{(K-1) K}\right]
$$

for the $\Pi_{k} \mathrm{~s}$ of (3). For the $K$-sided die, $\Lambda_{k}=\Pi_{k}$. Equation (4) can be thought of as inverting Born's rule, that is, we can write down the state $\rho$ that will give rise to the probabilities $p_{k}$ via Born's rule for the SIC-POM. The set of probabilities $\left\{p_{k}\right\}$ thus provides a complete description of the state $\rho$-this is the sense in which the SIC-POM is informationally complete. We will often use the notation $\rho \sim\left\{p_{k}\right\}$ to denote this relation. We will also occasionally use simply $p$ to denote the list $\left\{p_{1}, p_{2}, \ldots, p_{K}\right\}$. Any set of probabilities $\left\{p_{k}\right\}$ is always understood to satisfy $p_{k} \geq$ 0 for all $k$ and $\sum_{k} p_{k}=1$, and we sometimes refer to the set as a probability distribution.

The goal of a tomographic problem, classical or quantum, is to provide a reasonable estimator for the true state $\rho$, given the data from performing a chosen IC-POM on every one of $N$ identical copies of the input state. To be concrete, in the subsequent analysis, we represent the data from the $N$ measurements as a sequence of clicks in the $K$ possible detectors: $D_{N} \equiv\left\{c_{1}, c_{2}, \ldots, c_{N}\right\}$, where $c_{l} \in\{1,2, \ldots, K\}$ is the detector that clicked in the $l$ th measurement. We can summarize the data by collecting together the number of clicks for each detector: $\left\{n_{1}, n_{2}, \ldots, n_{K}\right\}$, where $n_{k}$ is the number of times the $k$ th detector clicked in the $N$ measurements. We will use the notation $D_{N} \sim\left\{n_{1}, n_{2}, \ldots, n_{K}\right\}$ to refer to the summary of a particular sequence of measurement outcomes. Note that the data must satisfy $\sum_{k=1}^{K} n_{k}=N$.

A point estimator $\hat{\rho}$ is a map from the set $\mathcal{D} \equiv\left\{\mathcal{D}_{1}, \mathcal{D}_{2}, \ldots, \mathcal{D}_{N}, \ldots\right\}$ of all possible data to the set $\mathcal{S}$ of all possible (physical) states. Here, $\mathcal{D}_{N}$ denotes all possible measurement outcomes on $N$ copies of the state. For the classical die problem, for example, $\mathcal{D}_{N}$ consists of all possible sequences of faces revealed in $N$ tosses. The set $\mathcal{S}$ consists of all states $\rho=\sum_{k} p_{k} \Lambda_{k}$ where $\left\{p_{k}\right\}$ is a probability distribution. We denote the point estimator for data $D_{N}$ as $\hat{\rho}\left(D_{N}\right)$, and denote the set of all point estimators, that is, all maps $\hat{\rho}: \mathcal{D} \rightarrow \mathcal{S}$, by $\hat{\mathcal{S}}$.

${ }^{b}$ Here, we take the liberty to include the classical case under the general name of SIC-POM. In the classical case, the SIC-POM is simply a projective measurement-a von Neumann measurement. A projective measurement is IC for the classical case, though not for the quantum case. 


\subsection{The maximum-likelihood estimator}

A very popular approach to a point estimator is the maximum-likelihood method. The ML method prescribes as the point estimator the state at which the likelihood function for the observed data attains its maximum. The likelihood function - the probability that the state $\rho \sim\left\{p_{k}\right\}$ gives rise to the data $D_{N}$-is

$$
\mathcal{L}\left(D_{N} \mid \rho\right)=\prod_{k=1}^{K} p_{k}^{n_{k}}
$$

To find the ML estimator for the classical die problem, we solve the following constrained maximization problem:

$$
\begin{array}{ll} 
& \max _{\rho \sim\left\{p_{k}\right\}} \mathcal{L}\left(D_{N} \mid \rho\right), \\
\text { subject to } \quad \sum_{k} p_{k}=1 \quad \text { with } \quad p_{k} \geq 0 \text { for all } k .
\end{array}
$$

This gives the ML estimator $\hat{\rho}_{\mathrm{ML}} \equiv \sum_{k}\left(\hat{p}_{k}\right)_{\mathrm{ML}} \Pi_{k}$ with

$$
\left(\hat{p}_{k}\right)_{\mathrm{ML}}=\frac{n_{k}}{N} \equiv \nu_{k} \text {. }
$$

For large $N$, the ML estimator for the classical die is intuitive: From a frequentist's perspective, the long-run $(N \rightarrow \infty)$ relative frequencies $\nu_{k}$ should approach the probabilities $p_{k}$. What about small $N$ ? Suppose a coin (a "2-sided die") is tossed just once, and gives "heads". Hardly anyone will put his money on the estimator $\hat{p}_{k}=n_{k} / N$, which means setting $\hat{p}_{\text {head }}=1$, and $\hat{p}_{\text {tail }}=0$. This lack of confidence in the estimator is well justified if one considers the fact that, for $D_{1} \sim\{1,0\}$, the likelihood function is not very sharply peaked at $p_{\text {head }}=1$, and $p_{\text {head }}$ values near 1 have similar likelihood. Suppose we make more tosses, and always get heads. Then, we gain confidence in the estimator $\hat{p}_{\text {heads }}=1$ and $\hat{p}_{\text {tails }}=0$, as is reflected by the likelihood function getting more and more sharply peaked at $p_{\text {head }}=1$; see Fig. 1 .

Observe that the ML estimator in (8) depends only on the relative frequencies $\nu_{k}$, and not on $N$, the total number of tosses made. For the above example where $D_{N} \sim\{N, 0\}$, the ML estimator is always $\hat{p}_{\text {heads }}=1$ and $\hat{p}_{\text {tails }}=0$ for all $N$. Only the confidence (loosely quantified by the width of the likelihood function) in the estimator changes with $N$. In many situations, only the point estimator, and not the confidence interval associated with it, is carried forward into subsequent analysis. However, a statement that $\hat{p}_{\text {head }}=1$ if $N=1$ is clearly not of the same standing as saying $\hat{p}_{\text {head }}=1$ after $N=10,000$ tosses. This invites us to look for a point estimator that itself reflects our changing level of confidence as $N$ changes.

Another peculiarity of the ML estimator is visible in Fig. 1 The point estimator is reported as a point on the boundary of the allowed values for $p_{\text {head }}$. This corresponds to the statement that tails can never occur. In general, rank deficiency in the estimator - that is, there exists at least one pure state $|\psi\rangle$ on which $\hat{\rho}_{\mathrm{ML}}$ has no support, $\left\langle\psi\left|\hat{\rho}_{\mathrm{ML}}\right| \psi\right\rangle=0$ - says that a detector that projects into the rank-deficient sector can never click, a statement that cannot be justified with finite $N$. Yet, the 


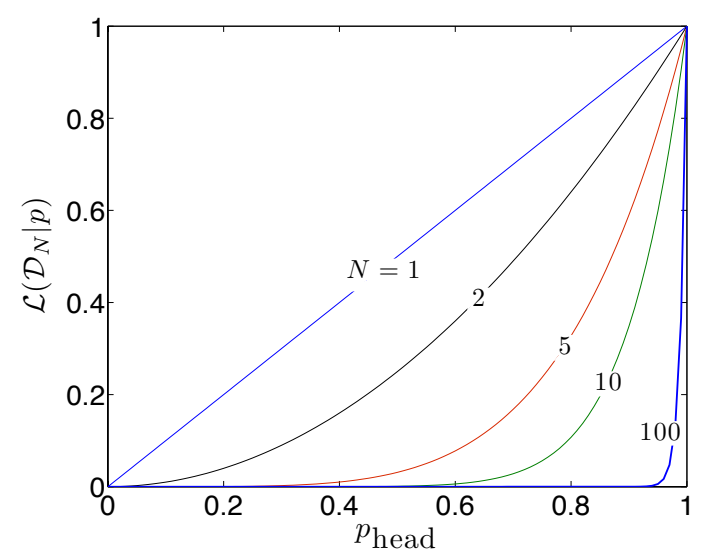

Fig. 1. Likelihood function for a 2-sided die (coin) with data $D_{N} \sim\{N, 0\}$, for $N=1,2,5,10$ and 100 .

ML estimator is frequently rank-deficient whenever $N$ is of the order of $K$, such that there is non-negligible probability that at least one of the detectors has no clicks. This invites us to look for a point estimator that is full-rank for all finite $N$.

\subsection{Mean estimators}

An alternative to the ML estimator that takes $N$ into account is suggested by Fig. 1 For small $N$, the likelihood is significant for a large region of $p_{\text {head }}$ values around the maximum; for large $N$, the likelihood rapidly drops as we move away from the maximum. This suggests using the likelihood function as a weight to construct a point estimator. We weigh each state $\rho$ of the system by its likelihood, given data $D_{N}$, and perform an average over all states to obtain the mean estimator

$$
\hat{\rho}_{\mathrm{ME}}\left(D_{N}\right) \equiv \frac{\int \mathrm{d} \phi(\rho) \mathcal{L}\left(D_{N} \mid \rho\right) \rho}{\int \mathrm{d} \phi(\rho) \mathcal{L}\left(D_{N} \mid \rho\right)},
$$

where $\mathrm{d} \phi$ is an integration measure that tells us how to perform a sum over states; $\mathrm{d} \phi$ should be non-negative on all physical states of the system, and zero elsewhere. We can require, in addition, that $\int \mathrm{d} \phi(\rho)=1$ for interpretation of $\mathrm{d} \phi$ as a probability distribution. This is, however, not necessary and we only require that $\mathrm{d} \phi$ is not too pathological, so that the integrals in (9) exist.

A reader familiar with Bayesian methods will recognize that the use of a prior distribution $\mathrm{d} \mu(\rho)$, which encapsulates the experimenter's prior information about the probability of occurrence of each state $\rho$, fits within this framework of mean estimators. If one chooses $\mathrm{d} \phi=\mathrm{d} \mu$, then by Bayes's theorem, $\mathrm{d} \phi(\rho) \mathcal{L}\left(D_{N} \mid \rho\right)$ is proportional to the posterior distribution $\mathrm{d} \mu\left(\rho \mid D_{N}\right)$, that is, the probability for 
state $\rho$ given data $D_{N}$. The mean estimator for this choice of $\mathrm{d} \phi$ is then simply the mean state for the posterior distribution. This particular mean estimator has a long history in Bayesian estimation, and is also sometimes used in the quantum literature.

For us, the integration measure $\mathrm{d} \phi$ need not be chosen to represent our prior information about the identity of the true state, but is a functional parameter that we can adjust to satisfy desired optimality conditions. By varying $\mathrm{d} \phi$, we can describe a reasonable class of point estimators - the class of mean estimatorsconstructed as in (9), that is,

$$
\hat{\mathcal{S}}_{\mathrm{ME}} \equiv\left\{\hat{\rho}_{\mathrm{ME}}[\mathrm{d} \phi]\right\}
$$

For the case of a $K$-sided die, the mean estimator can be written more explicitly as

$$
\begin{aligned}
& \hat{\rho}_{\mathrm{ME}} \equiv \sum_{k=1}^{K}\left(\hat{p}_{k}\right)_{\mathrm{ME}} \Lambda_{k}, \\
& \text { with }\left(\hat{p}_{k}\right)_{\mathrm{ME}} \equiv \frac{\int_{0}^{1}(\mathrm{~d} p) \delta\left(1-\sum_{l=1}^{K} p_{l}\right) f(p) \mathcal{L}\left(D_{N} \mid p\right) p_{k}}{\int_{0}^{1}(\mathrm{~d} p) \delta\left(1-\sum_{l=1}^{K} p_{l}\right) f(p) \mathcal{L}\left(D_{N} \mid p\right)} .
\end{aligned}
$$

Here, we parameterize the states of the die by their probabilities $\left\{p_{k}\right\}$. The integration measure is written explicitly as $\mathrm{d} \phi(\rho)=(\mathrm{d} p) \delta\left(1-\sum_{l=1}^{K} p_{l}\right) f(p)$, where $(\mathrm{d} p)$ denotes the volume element $\mathrm{d} p_{1} \mathrm{~d} p_{2} \ldots \mathrm{d} p_{K}$, the delta function enforces $\sum_{l} p_{l}=1$, and $f(p)$ is a non-negative function that we can choose to suit our needs.

A natural symmetry in the $K$-sided die problem lies in the labeling of the different faces as $1,2, \ldots, K$ : A permutation of these arbitrary labels does not change the physical description of the die. This symmetry should be reflected in the choice of $f(p)$ as an invariance under permutation of the label $k$. A particularly simple choice of $f$ with this invariance is

$$
f(p)=\left(\prod_{k=1}^{K} p_{k}\right)^{\beta-1} \quad \text { with } \beta>0 .
$$

The resulting mean estimator for this choice of $f$ is

$$
\left(\hat{p}_{k}\right)_{\mathrm{ME}}=\frac{n_{k}+\beta}{N+K \beta} \text {. }
$$

There is an alternate way of arriving at this estimator following ML ideas. Suppose we obtained data $D_{N} \sim\left\{n_{1}, n_{2}, \ldots n_{k}, \ldots, n_{K}\right\}$. We add "fake counts" to every detector, of an amount $\beta$, so that the data becomes $D_{N, \beta} \sim\left\{n_{1}+\beta, n_{2}+\right.$ $\left.\beta, \ldots, n_{k}+\beta, \ldots, n_{K}+\beta\right\}$ and the total number of counts appears to be $N+K \beta$. Then, the ML estimator for this modified data is exactly that given in (13). This 
estimator is sometimes referred to as the "add- $\beta$ " estimator, and is used as an adhoc procedure to avoid reporting an ML estimator that lies on the boundary. Note that the $\beta \rightarrow 0$ limit of (13) corresponds exactly to the ML estimator of (8), but one cannot use the $\beta=0$ version of 12 in (11).

\subsection{Assessing the quality of an estimation procedure}

How well does a particular estimation procedure perform? To answer this question, we need to define a figure-of-merit that quantifies how far from the true state an estimator is. Given data $D_{N}$, we compute the error in our guess $\hat{\rho}\left(D_{N}\right)$ of the true state,

$$
\text { estimation error } \equiv E\left(\hat{\rho}, \rho, D_{N}\right) \equiv \operatorname{dist}\left(\hat{\rho}\left(D_{N}\right), \rho\right),
$$

where $\operatorname{dist}(\hat{\rho}, \rho)$ is often chosen to be a formal distance between two states $\rho$ and $\hat{\rho}$, like the trace distance or the Euclidean distance. More generally, it is a function that assigns a (non-negative) "cost" whenever $\rho \neq \hat{\rho}$.

Of course, we are not always going to get the data $D_{N}$ every time we perform tomography. Instead, one should assess the efficacy of the estimation procedure for $\rho$ by averaging the estimation error over all possible data $D_{N}$ that one could have obtained. Using terminology standard in estimation theory (see, for example, Ref. 11), this gives the risk

$$
R_{N}(\hat{\rho}, \rho) \equiv \sum_{D_{N} \in \mathcal{D}_{N}} \mathcal{L}\left(D_{N} \mid \rho\right) E\left(\hat{\rho}, \rho, D_{N}\right)
$$

The risk $R_{N}(\hat{\rho}, \rho)$ still only tells us how good the estimator $\hat{\rho}$ is for a given true state $\rho$. But, we have to judge the merits of an estimation procedure while not knowing the identity of the true state (hence the need for tomography). If there exists a $\hat{\rho}$ such that the risk $R_{N}(\hat{\rho}, \rho)$ for all true states $\rho$ is smaller than that of any other estimation procedure, this $\hat{\rho}$ will clearly be the best procedure to use. However, an estimator with such miraculous properties is not likely to exist.

Instead, suppose we only ask that the estimator performs well "on average" over the true states. For example, a $\hat{\rho}$ that gives a large risk for a particular state $\rho_{0}$ but small risk values for all other true states can be considered a good estimation procedure as long as the probability that $\rho_{0}$ is indeed the true state is tiny compared

to other states. This requires some knowledge about the probability distribution of the true states, that is, the prior distribution $\mathrm{d} \mu(\rho)$. If we know the prior distribution, a figure-of-merit that can be used to assess an estimation procedure is its average performance over the true states, that is, the risk weighted by the prior distribution,

$$
F_{N}(\hat{\rho}, \mathrm{d} \mu) \equiv \int \mathrm{d} \mu(\rho) R_{N}(\hat{\rho}, \rho)
$$

We refer to $F_{N}$ as the average risk. This includes the case where one does know the identity of the true state to be some state $\tau$ : $\mathrm{d} \mu(\rho)=\mathrm{d} \rho \delta(\rho-\tau)$, so that 
$F_{N}(\hat{\rho}, \mathrm{d} \mu)=R_{N}(\hat{\rho}, \tau)$. The case where $\hat{\rho}$ performs poorly only on a single state $\rho_{0}$ out of a possible (discrete) set of states $\mathcal{S} \equiv\left\{\rho_{i}\right\}_{i=0}^{L}$ involves using the prior $\mu\left(\rho_{i}\right)=q_{i}$ for $\rho_{i} \in \mathcal{S}$ and 0 otherwise, with $q_{0} \ll q_{i \neq 0}$. A large risk for $\rho_{0}$ is suppressed in $F$ by a small enough value of $q_{0}$.

Given a prior distribution, the average risk quantifies the efficacy of the estimator $\hat{\rho}$. To find the best estimation procedure among the set $\hat{\mathcal{S}}$ of all possible $\hat{\rho} \mathrm{s}$, one minimizes the average risk:

$$
\min _{\hat{\rho} \in \hat{\mathcal{S}}} F_{N}(\hat{\rho}, \mathrm{d} \mu) \quad \text { (Bayes). }
$$

An estimator (not necessarily unique) that minimizes the average risk is known as a Bayes estimator for the prior distribution $\mathrm{d} \mu$. Note that this requirement of choosing a prior to assess the efficacy of an estimation procedure in terms of average risk applies even for schemes like ML methods which, by themselves, do not require a choice of prior or integration measure.

Bayes estimators are well studied in the state estimation literature. The following fact ${ }^{C}$ relates mean estimators to Bayes estimators, which we will find useful later (for a self-contained proof of this fact, see Appendix B):

Fact 1. Suppose we choose the square of the Euclidean distance - the squared error - to define the estimation error:

$$
E\left(\hat{\rho}, \rho, D_{N}\right)=\operatorname{dist}\left(\hat{\rho}\left(D_{N}\right), \rho\right) \equiv \operatorname{tr}\left\{\left(\hat{\rho}\left(D_{N}\right)-\rho\right)^{2}\right\} .
$$

Then, the unique Bayes estimator for prior distribution $\mathrm{d} \mu$ is the mean estimator $\hat{\rho}_{\mathrm{ME}}[\mathrm{d} \mu]$.

The prior distribution $\mathrm{d} \mu(\rho)$ encapsulates our knowledge, not of the source at hand, but of the preparer of the source. Imagine that the preparer, say Alice, has promised to provide a source that puts out identical copies of a state $\rho$. Alice is, however, free to choose which particular state $\rho$ is. Our information about the probability that Alice provides us with a source that puts out state $\rho$ is given by $\mathrm{d} \mu(\rho)$. The data $D_{N}$ collected from measuring a single instance of the source provided by Alice cannot yield us any information about $\mathrm{d} \mu(\rho)$ (other than excluding states of the source that could not have given rise to $\left.D_{N}\right)$. $\mathrm{d} \mu(\rho)$ must reflect prior knowledge about the preparer gathered from previous interaction with different sources provided by Alice.

In most tomographic scenarios, such prior knowledge is absent, and it seems desirable to say "we don't know". Converting the heuristic notion of "we don't know" into a rigorous "uninformative prior" is, unfortunately, fraught with difficulties. For example, it would seem natural to assign equal probabilities to all states, in the absence of knowledge of which states are more probable. This is not a problem for a discrete set of states labeled by a discrete label $i$-it simply says that all the

${ }^{\mathrm{c}}$ This is Corollary 1.2 in Chapter 4 of Ref. 11 
$q_{i} \mathrm{~s}$ are equal. For a continuous set of states, which we parameterize using some continuous parameter $x$, equal probability of occurrence means setting $\mathrm{d} \mu(\rho)=d x$. However, there is no unique way of parameterizing the states, and equal probability in one parameterization in general does not translate into equal probability in a different parameterization. One often-used way to deal with this is to give up the idea of equal probabilities for all states, and ask for an uninformative prior with the property of parameterization invariance, for a relevant class of parameterization. An example is the Jeffreys prior (see, for example, Ref. 7, p. 181 for a discussion), which is scale-invariant, that is, invariant under reparameterization $x \rightarrow x^{m}$ for some power $m$.

\subsection{Minimaxity}

Assessing an estimator according to its average risk and using a Bayes estimator for $\mathrm{d} \mu$ only works well if the true distribution describing Alice the preparer is indeed $\mathrm{d} \mu$. Given that we do not usually know the prior distribution, and since even choosing something like an uninformative prior is far from straightforward, using a Bayes estimator for some choice of $\mathrm{d} \mu$ seems poorly justified. Minimax approaches offer a way out of this.

Instead of using the average risk as a figure-of-merit, an alternative is to use the worst-case risk, that is, the maximum risk (over all possible true states) of using estimator $\hat{\rho}$. This does away with the requirement of choosing a prior distribution to perform the averaging of the risk. The best estimator is found by minimizing the worst-case risk:

$$
\min _{\hat{\rho} \in \hat{\mathcal{S}}} \max _{\rho} R_{N}(\hat{\rho}, \rho) \quad(\operatorname{minimax}) .
$$

An estimator (not necessarily unique) that minimizes the worst-case risk is known as a minimax estimator.

Carrying out this double optimization to find a minimax estimator is, of course, non-trivial. There is, however, a fact ${ }^{\mathrm{d}}$ that can sometimes simplify the search for a minimax estimator (see Appendix C for a self-contained proof):

Fact 2. An estimator with constant risk that is also a Bayes estimator for some prior distribution $\mathrm{d} \mu$ is a minimax estimator. If the estimator is also the unique Bayes estimator for some $\mathrm{d} \mu$, then it is the unique minimax estimator.

Relating minimaxity to Bayes estimators is useful because much more is known about Bayes estimators than minimax estimators. For example, Facts 1 and 2 tell us that in scenarios where the squared error is the suitable figure-of-merit, the unique minimax estimator can be found by looking for a mean estimator $\hat{\rho} \in \hat{\mathcal{S}}_{\mathrm{ME}}$ with constant risk (if it exists). We will make use of this in the next section.

${ }^{\mathrm{d}}$ This is Corollary 1.5 in Chapter 5 of Ref. 11 . 


\subsection{The minimax estimator for the $K$-sided die}

For tomography with SIC-POMs, the squared error has the simple form

$$
\operatorname{tr}\left\{\left(\hat{\rho}\left(D_{N}\right)-\rho\right)^{2}\right\}=\frac{(K-1) K}{(d-1) d} \sum_{k}\left(\hat{p}_{k}-p_{k}\right)^{2}
$$

where $\hat{\rho} \equiv \sum_{k} \hat{p}_{k} \Lambda_{k}$ and $\rho \equiv \sum_{k} p_{k} \Lambda_{k}$. The squared error is proportional to the sum of squares of the difference in the probabilities $\left\{\hat{p}_{k}\right\}$ and $\left\{p_{k}\right\}$. The corresponding risk is thus nothing more than the mean squared error (MSE) commonly used in classical state estimation.

It is easy to work out the expression for the MSE for the class of mean estimators for the $K$-sided die given in (13). In particular, there exists a special value of $\beta$ (as used in (13)) such that the MSE is independent of $p$, that is, constant over all states,

$$
\beta=\frac{\sqrt{N}}{K}
$$

Using Facts 1 and 2 , we know that the mean estimator given in 13 with this value of $\beta$ is also the unique minimax estimator for the $K$-sided die problem, with the choice of the squared error as the figure-of-merit. This minimax property justifies objectively the choice of the integration measure $\mathrm{d} \phi \equiv(\mathrm{d} p) f(p)=(\mathrm{d} p)\left(\prod_{k} p_{k}\right)^{\beta-1}$ with $\beta=\sqrt{N} / K$.

For this choice of $\beta$, we can write the minimax estimator $\hat{\rho}_{\mathrm{MM}} \equiv \sum_{k}\left(\hat{p}_{k}\right)_{\mathrm{MM}} \Pi_{k}$ for the $K$-sided die in a form that exhibits its structure clearly,

$$
\begin{aligned}
\left(\hat{p}_{k}\right)_{\mathrm{MM}} & =\frac{1}{K} a_{N}+\nu_{k} b_{N}, \\
\text { with } \quad a_{N} & \equiv \frac{1}{1+\sqrt{N}}, \quad b_{N} \equiv \frac{1}{1+1 / \sqrt{N}} .
\end{aligned}
$$

The parameters $a_{N}$ and $b_{N}$ depend only on $N$ and satisfy the relation $a_{N}+b_{N}=1$. Observe that $a_{N}$ approaches zero as $N$ gets large, while $b_{N}$ approaches unity, for which the minimax estimator approaches the ML estimator $\left(\hat{p}_{k}\right)_{\mathrm{ML}}=\nu_{k}$. For $N$ small, $a_{N}$ is significant, and the two estimators differ.

Observe that, unlike the ML estimator, this minimax estimator is always fullrank for finite $N$, since for any pure state $|\psi\rangle$ of the system,

$$
\left\langle\psi\left|\hat{\rho}_{\mathrm{MM}}\right| \psi\right\rangle=\frac{1}{K} a_{N}+b_{N} \sum_{k} \nu_{k}|\langle\psi \mid k\rangle|^{2}>0 .
$$

We can also compute the purity of the minimax estimator,

$$
\begin{aligned}
\operatorname{tr}\left\{\hat{\rho}_{\mathrm{MM}}^{2}\right\} & =\sum_{k=1}^{K}\left(\hat{p}_{k}\right)_{\mathrm{MM}}^{2}=\frac{1}{K}+b_{N}^{2}\left(\sum_{k} \nu_{k}^{2}-\frac{1}{K}\right) \\
& \leq 1-\left(1-\frac{1}{K}\right)\left(1-b_{N}^{2}\right),
\end{aligned}
$$


which is strictly less than 1 for finite $N$. The equality is attained when the data is such that all clicks are in a single detector. Having the purity bounded away from 1 is immediately obvious from the fact that the estimator is always full-rank. However, the expression for the purity reveals more interesting features. For $K$ fixed, as $N$ increases, the bound on the purity increases towards 1 , and the estimator can approach a pure state, expressing our increasing confidence in claiming a definite pure state as we gather more data. Also, for $N$ fixed, the purity of the estimator decreases as $K$ increases. This is also intuitive: If $K$ is large, we would require more data to convince ourselves that certain detectors will never click.

The minimax estimator for the $K$-sided die problem circumvents both complaints we had about the ML estimator. The minimax estimator itself has a dependence on $N$, and furthermore is never rank-deficient for finite $N$. In the remainder of this paper, we would like to adapt this minimax estimator to the quantum problem, while still retaining these two desirable properties.

\section{The quantum problem}

In this section, we turn to the tomography of a quantum system. We begin by pointing out the differences between the classical and the quantum problems (Sections 3.1 and 3.2 . These considerations provide clues to adapting the minimax estimator of the classical die problem to the quantum context (Sections 3.3 and 3.4 ).

\subsection{SIC-POM for a quantum system}

In moving from the classical to the quantum problem, the first difference we meet is that the IC-POM that one can perform on the quantum system for full tomography is non-unique. This has to do with the fact that there is no unique preferred basis such that all quantum states are diagonal in that basis. We can, however, still choose to make use of a SIC-POM which offers efficiency advantages over other choices of IC-POM ${ }^{12}$ Related to the lack of a unique preferred basis is the fact that, unlike the classical case, the POM outcomes of a SIC-POM are no longer mutually orthogonal: $\operatorname{tr}\left\{\Pi_{k} \Pi_{l}\right\} \neq 0$ for $k \neq l$. According to Appendix A, a SICPOM for a quantum system has $K=d^{2}$, and the $\Lambda_{k}$ operators of (5) take the simple form of $\Lambda_{k}=d(d+1) \Pi_{k}-1$. In our discussion below, we will only consider such a SIC-POM for tomography of a quantum system.

For a single qubit, that is, a two-dimensional quantum system, the SIC-POM is the tetrahedron measurement, $\frac{13}{13}$ with $\mathrm{POM}$ outcomes proportional to projectors onto the legs of a regular tetrahedron inscribed within the Bloch sphere. The tetrahedron measurement is non-unique in that the orientation of the tetrahedron within the Bloch sphere is left to the choice and convenience of the experimenter. Nevertheless, given a particular orientation, the POM outcomes of the tetrahedron measurement can be written in terms of the Pauli vector operator $\vec{\sigma} \equiv\left(\sigma_{x}, \sigma_{y}, \sigma_{z}\right)$ 
as

$$
\Pi_{k}=\frac{1}{4}\left(1+\vec{a}_{k} \cdot \vec{\sigma}\right), \quad k=1,2,3,4,
$$

where each $\vec{a}_{k}$ is one of the four legs of the tetrahedron. The tetrahedron vectors $\vec{a}_{k}$ satisfy $\vec{a}_{k} \cdot \vec{a}_{l}=\frac{4}{3} \delta_{k l}-\frac{1}{3}$. Their linear dependence is captured by the facts that they sum to zero, $\sum_{k} \vec{a}_{k}=0$, and are complete, $\frac{3}{4} \sum_{k} \vec{a}_{k} \vec{a}_{k}=\overleftrightarrow{1}$. The probability of obtaining the $k$ th outcome $\Pi_{k}$ for a qubit state $\rho=\frac{1}{2}(1+\vec{s} \cdot \vec{\sigma})$ is given by $p_{k} \equiv \operatorname{tr}\left\{\rho \Pi_{k}\right\}=\frac{1}{4}\left(1+\vec{a}_{k} \cdot \vec{s}\right)$, and $\Lambda_{k}=6 \Pi_{k}-1$.

Before we describe the quantum problem further, let us make a side remark regarding the choice of figure-of-merit. For the classical die problem, while the state of the die can be gathered into a single operator $\rho=\sum_{k} p_{k} \Lambda_{k}, \rho$ is but a book-keeping device for the probabilities $\left\{p_{k}\right\}$ one is truly concerned with. The squared error, which directly measures how much the estimated probabilities differ from the true probabilities, is thus a natural way to quantify the estimation error. Of course, one can use the Euclidean distance (rather than its square), but taking the square has analytical advantages. One just needs to note that doubling the difference in probabilities quadruples the squared error.

For the quantum problem, however, things are different. If the purpose of the quantum tomography is to predict the outcome of a future measurement of the same SIC-POM used to perform tomography, then the $p_{k} \mathrm{~s}$ are again the only quantities of relevance, and the use of the squared error is, as in the classical case, rather natural. However, if one's goal for tomography is to predict outcomes of a different measurement that can yield information complementary to that provided by the tomographic SIC-POM, then quantifying the estimation error in terms of differences in the probabilities $\left\{p_{k}\right\}$ may not be suitable. Instead, one might choose to use, for example, the fidelity or the trace distance between the estimator and the true state $\mathrm{e}^{\mathrm{a}}$ Nevertheless, for calculational ease, in the remainder of the paper, we shall continue to use the squared error as our figure-of-merit.

The squared error is also the (square of the) Euclidean distance between the estimator and true state when viewed as vectors in the Hilbert-Schmidt space. We emphasize that there is no single figure-of-merit that is suitable for all situations, but it should be chosen in accordance with the task at hand.

\subsection{Physicality constraints}

Consider any probability distribution. Does $\left\{p_{k}\right\}$ always correspond to outcome probabilities that can be obtained by applying Born's rule for a SIC-POM to a physical state of the system? Equivalently, one can ask whether $\rho \equiv \sum_{k} p_{k} \Lambda_{k}$, for the SIC-POM we are considering, describes a physical state of the system, that is, $\rho$ has unit trace and is non-negative, for any probability distribution $\left\{p_{k}\right\}$.

${ }^{e}$ Note, however, that for the qubit problem, the square of the trace distance is equal to the squared error. 
To answer this question, let us examine the quantity $p^{2} \equiv \sum_{k} p_{k}^{2}$. Since $\sum_{k} p_{k}=1$, the minimum value of $p^{2}$ is attained when all the $p_{k} \mathrm{~s}$ are equal. This gives

$$
p^{2} \geq \frac{1}{K}
$$

true for any probability distribution $\left\{p_{k}\right\}$.

What about the maximum value of $p^{2}$ ? Any physical state, whether quantum or classical, must satisfy $\operatorname{tr}\left\{\rho^{2}\right\} \leq 1$. Using $\rho=\sum_{k} p_{k} \Lambda_{k}$, and writing $\Lambda_{k}=a \Pi_{k}+b$ for a SIC-POM ( $a$ and $b$ can be deduced from $(5)$ ), it is easy to show that $\operatorname{tr}\left\{\rho^{2}\right\} \leq 1$ implies $p^{2} \leq(1-b) / a$. A $K$-sided classical die problem has $a=1$ and $b=0$, which gives

$$
\frac{1}{K} \leq\left(p^{2}\right)_{\mathrm{K}-\text { sided die }} \leq 1
$$

This is satisfied for any probability distribution $\left\{p_{k}\right\}$. The physicality requirement that $\operatorname{tr}\left\{\rho^{2}\right\} \leq 1$ does not constrain the $p_{k}$ s further. In fact, for the classical problem, $\rho=\sum_{k} p_{k} \Lambda_{k}$ is physical for any probability distribution.

For the quantum problem with a SIC-POM, however, we have a different situation. In this case, $a=d(d+1)$ and $b=-1$, which gives

$$
\frac{1}{K} \leq\left(p^{2}\right)_{\text {quantum }} \leq \frac{2}{d(d+1)}
$$

The right side of the inequality is strictly less than 1 for $d>1$. For example, the qubit problem with the tetrahedron measurement has the physicality constraint

$$
\frac{1}{4} \leq\left(p^{2}\right)_{\text {qubit }} \leq \frac{1}{3}
$$

Only probability distributions $\left\{p_{k}\right\}$ that obey 28 can correspond to a physical quantum state. For example, $\left\{p_{1}=1, p_{2,3,4}=0\right\}$ does not correspond to a physical qubit state. This is a direct manifestation of the non-orthogonality of the POM outcomes comprising a quantum SIC-POM. Note that 29 is also sufficient for the qubit problem: Any probability distribution $\left\{p_{k}\right\}$ satisfying 29 corresponds to a physical qubit state. For higher-dimensional quantum system, there are additional physicality constraints, apart from 28 , that $\left\{p_{k}\right\}$ must satisfy.

Additional physicality constraints on $\left\{p_{k}\right\}$ mean that the expression for the mean estimator for a quantum state is not just the expression for the classical die problem given in (11). For example, in the qubit problem, the mean estimator is 
now

$$
\begin{aligned}
& \hat{\rho}_{\mathrm{ME}} \equiv \sum_{k=1}^{K}\left(\hat{p}_{k}\right)_{\mathrm{ME}} \Pi_{k} \\
& \text { with } \quad\left(\hat{p}_{k}\right)_{\mathrm{ME}} \equiv \frac{\int_{0}^{1}(\mathrm{~d} p) \delta\left(1-\sum_{l=1}^{4} p_{l}\right) \eta\left(\frac{1}{3}-p^{2}\right) f(p) \mathcal{L}\left(D_{N} \mid p\right) p_{k}}{\int_{0}^{1}(\mathrm{~d} p) \delta\left(1-\sum_{l=1}^{4} p_{l}\right) \eta\left(\frac{1}{3}-p^{2}\right) f(p) \mathcal{L}\left(D_{N} \mid p\right)} .
\end{aligned}
$$

Here, $\eta($ ) is Heaviside's unit step function: $\eta(x)=0$ if $x<0$, and $\eta(x)=1$ if $x>0$. The step function enforces the upper bound in 29 . With this expression, one can define an optimization procedure over all possible $f(p)$ functions to look for a minimax (for example, using the MSE as the risk) estimator for the qubit. This is, of course, difficult to perform. What would be simpler, is an $f(p)$ for which the MSE is constant for all qubit states, which would then give a minimax estimator according to Facts 1 and 2 . Unfortunately, our preliminary attempts at this yielded a function $f(p)$ that flies in the face of common sense.

\subsection{Adapting the classical minimax estimator to the quantum problem}

Instead of tackling the difficult problem of finding a minimax estimator, we can try to build a simple estimator for quantum states by adapting the minimax estimator from the classical die problem. For the quantum problem, the ML estimator has the same problem of having no dependence on $N$ as well as suffering from rank deficiency. The ML estimator for the quantum case tells us to report, as the point estimator, the physical quantum state at which the likelihood function attains its maximum. For the qubit problem discussed above, this corresponds to looking for the maximum of $\mathcal{L}\left(D_{N} \mid \rho\right)$ subject not only to the usual constraint of $\sum_{k} p_{k}=1$, but also the additional constraint that $\sum_{k} p_{k}^{2} \leq \frac{1}{3}$. Whenever the data $D_{N}$ are such that $\sum_{k} \nu_{k}^{2} \leq \frac{1}{3}$, the ML estimator is unmodified from the classical case: $\left(\hat{p}_{k}\right)_{\mathrm{ML}}=\nu_{k}$; when the inequality is violated, the ML estimator gives a state on the boundary of the Bloch sphere (that is, a rank-deficient state) such that $\sum_{k}\left(\hat{p}_{k}\right)_{\mathrm{ML}}^{2}=\frac{1}{3}$. The goal will be to adapt the minimax estimator from the classical die problem in such a way that we arrive at an estimator that has a reasonable dependence on $N$ and does not suffer from rank-deficiency.

For the moment, let us put aside the desire for a full-rank estimator, and focus on establishing a point estimator that is always physical for the quantum problem. Suppose we begin with the expression for the $\left(\hat{p}_{k}\right)_{\mathrm{MM}} \mathrm{s}$ for the $K$-sided die problem in 22 , which we rename here as

$$
\hat{p}_{k, 0} \equiv \frac{1}{K} a_{N}+\nu_{k} b_{N}
$$


We use these probabilities to construct an estimator for the quantum problem with a $K$-outcome SIC-POM in accordance with (4),

$$
\hat{\rho}_{0} \equiv \sum_{k} \hat{p}_{k, 0} \Lambda_{k}
$$

We emphasize that the $\Lambda_{k}$ s in $(32)$ are for the quantum SIC-POM.

Let us examine the quantity $p^{2}$ for $\left\{\hat{p}_{k, 0}\right\}$, which we will denote as $\hat{p}_{0}^{2}$. This was computed previously in (24), which can be rewritten as

$$
\hat{p}_{0}^{2} \leq \frac{2}{d(d+1)}\left\{1+\frac{d-1}{2 d}\left[\frac{(d+1)^{2}}{(1+1 / \sqrt{N})^{2}}-1\right]\right\} .
$$

Observe that this inequality is weaker, for $N \geq 1$ than the physicality constraint $\hat{p}_{0}^{2} \leq \frac{2}{d(d+1)}$ in 28 , necessary for $\hat{\rho}_{0}$ to be a physical state. This means that there always exist data sets (for example, all but one detector have zero clicks) for which $\hat{\rho}_{0}$ is not physical and, therefore, fails to be a valid estimator for the quantum problem.

Nevertheless, observe that data such that all but one detector have zero clicks occur only with a small probability for values of $N$ that are not too small. If the minimax estimator is physical for most data that we are likely to encounter, then there is some hope that this estimator can still work well for quantum states. After all, the quantum problem with a $K$-outcome SIC-POM looks very similar-from the perspective of the outcome probabilities - to the classical $K$-sided die problem as long as we are away from the boundary where the physicality constraints come into play.

Suppose we perform a "correction" to the estimator $\hat{\rho}_{0}$ whenever it is unphysical, by admixing just enough of the maximally mixed state to make the overall mixture physical. We take this new mixture to be the estimator

$$
\begin{aligned}
& \hat{\rho}=(1-\lambda) \hat{\rho}_{0}+\frac{\lambda}{d} \equiv \sum_{k} \hat{p}_{k} \Lambda_{k}, \\
& \text { with } \quad \hat{p}_{k} \equiv(1-\lambda) \hat{p}_{k, 0}+\frac{\lambda}{d^{2}},
\end{aligned}
$$

where $\lambda \geq 0$ is to be chosen as small as possible such that $\hat{\rho}$ is a physical state. For the qubit problem, one can be more explicit: If $\hat{\rho}_{0}$ is a physical qubit state, $\lambda=0$; otherwise, $\lambda$ is chosen such that $\hat{p}^{2} \equiv \sum_{k} \hat{p}_{k}^{2}=\frac{1}{3}$. It follows that $\lambda$, for the qubit problem, is

$$
\lambda=\eta\left(\sum_{k} \nu_{k}^{2}-\left(\sum \nu^{2}\right)_{\text {phy }}\right)\left[1-\sqrt{\frac{\left(\sum \nu^{2}\right)_{\text {phy }}-\frac{1}{4}}{\sum_{k} \nu_{k}^{2}-\frac{1}{4}}}\right] .
$$

Here, $\left(\sum \nu^{2}\right)_{\text {phy }}$ is the largest value of $\sum_{k} \nu_{k}^{2}$ such that the data will give a physical 
$\hat{\rho}_{0}$, that is,

$$
\sum_{k} \nu_{k}^{2} \leq \frac{1}{4}+\frac{1}{12 b_{N}^{2}} \equiv\left(\sum \nu^{2}\right)_{\text {phy }}
$$

as implied by (24).

Equation (34) provides a simple prescription for converting any estimator $\hat{\rho}_{0}$ from classical problems, not necessarily the minimax estimator we have used here, into an estimator for the quantum problem. For example, one can get a good approximation to the ML estimator this way. Suppose we ignore physicality constraints and look for the ML estimator for given data $D_{N}$ subject only to the constraints that the $p_{k} \mathrm{~s}$ are non-negative with unit sum. This is exactly the ML estimator if the problem is classical. We take this classical ML estimator for $\hat{\rho}_{0}$, and apply the prescription given in (34). This gives an estimator that is very close (for example, in terms of fidelity) to the one obtained from the ML scheme where one performs constrained maximization (taking physicality constraints into account) of the likelihood function.

To demonstrate the effectiveness of the estimator given in (34), we plot in Fig. 2 the maximum and minimum (over all possible input states) risks - measured using the MSE - for our estimator in the qubit case (labeled in Fig. 2 as 'min, max risk $\left.\left(\epsilon_{N}=0\right)^{\prime}\right)$. For comparison, we also plot the corresponding risk values for the ML estimator (labeled in Fig. 2 as 'min, max risk (ML)'). Observe that the maximum error for our estimator is significantly smaller than that for the ML estimator, indicating a step closer towards a true minimax estimator. For $N$ not too small, note also the much smaller difference between the maximum and minimum risk values for our qubit estimator as compared to the case for the ML estimator. This near-homogeneity of risk values over all states is inherited from the original classical minimax estimator that has constant risk. Risk homogeneity is attractive since it reflects an equal treatment of all input states, without having to implement the subjective construct of a uniform prior for a continuous set of states.

\subsection{Modifying the estimator to be full-rank-a minimax estimator}

In correcting the minimax estimator from the classical problem for physicality in the quantum case, we have lost the feature that the resulting estimator is always full-rank: Whenever $\hat{\rho}_{0}$ is unphysical, we correct it by choosing $\lambda$ just large enough to exactly cancel the most negative eigenvalue of $\hat{\rho}_{0}$, and so to give a non-negative state with (at least) one zero eigenvalue. In this section, we attempt to remedy this rank deficiency using a minimax approach.

Let us focus on the qubit problem with the tetrahedron measurement. We consider the same estimator as before in (34) with $d=2$. Now, rather than choosing $\lambda$ such that the physicality constraint on $\hat{p}^{2}$ is saturated $\left(=\frac{1}{3}\right)$, we choose $\lambda$ such that we saturate the constraint except for an overall factor of $\left(1-\epsilon_{N}\right)$, for some parameter $\epsilon_{N} \geq 0$. More precisely, we set $\lambda=0$ whenever $\hat{p}_{0}^{2} \leq \frac{1}{3}\left(1-\epsilon_{N}\right)$; otherwise, 


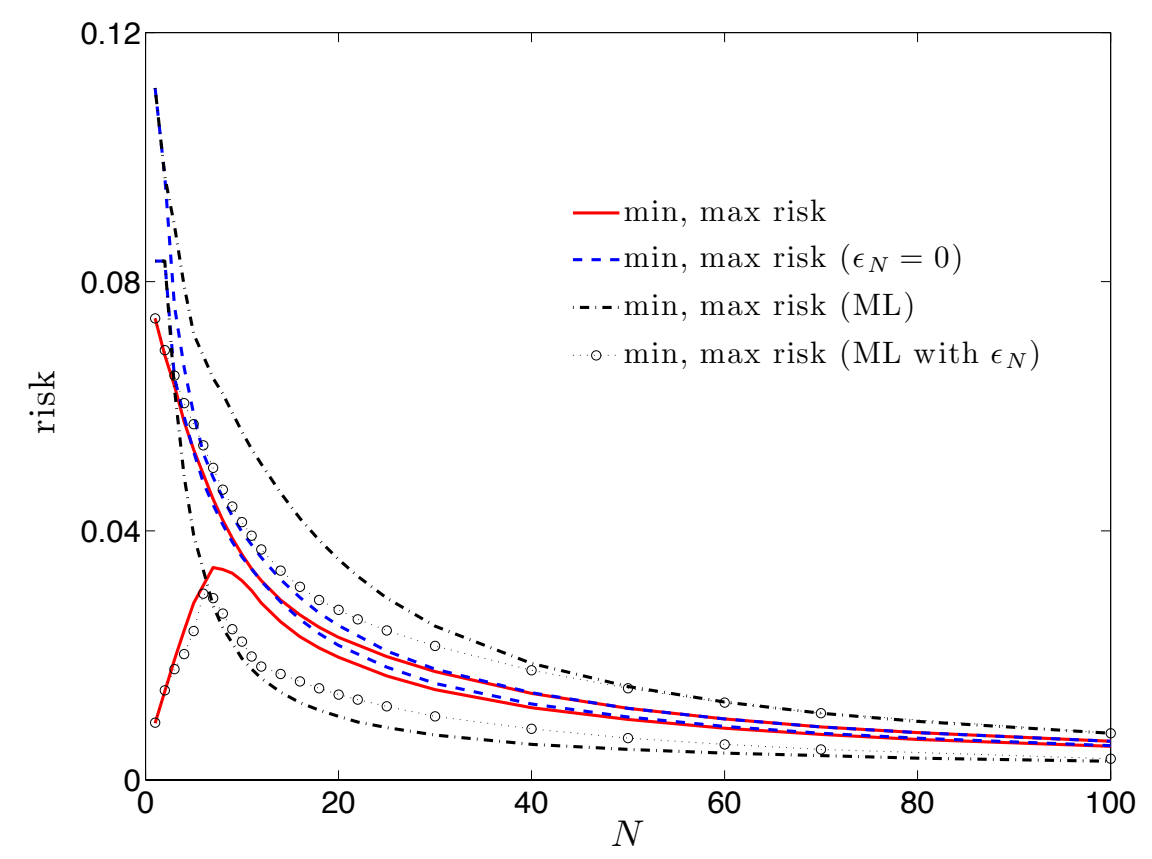

Fig. 2. The solid curves plot the minimum and maximum risk (over all qubit states) for the optimal value of $\epsilon_{N}$. The dash-dotted curves are the corresponding values for setting $\epsilon_{N}=0$ for all values of $N$. The dotted curves correspond to the risk values for the ML estimator, and the curves with a circular marker give risk values for ML modified by an $\epsilon_{N}$ parameter as described in the text.

$\lambda$ is chosen to ensure $\hat{p}^{2}=\frac{1}{3}\left(1-\epsilon_{N}\right)$. The latter case can be written more explicitly as an equation for $\lambda$,

$$
(1-\lambda)^{2} b_{N}^{2}\left(\sum_{k} \nu_{k}^{2}-\frac{1}{4}\right)=\frac{1-4 \epsilon_{N}}{12} .
$$

What remains is to choose the value of $\epsilon_{N}$. For this, we make use of a minimax procedure: Find the best value of $\epsilon_{N}$ by minimizing the worst-case risk, that is,

$$
\min _{\epsilon_{N} \geq 0} \max _{\rho} R_{N}(\hat{\rho}, \rho)
$$

where $\hat{\rho}$ is the estimator constructed from (34) with $\lambda$ chosen (when necessary) to satisfy (37). Equations (34) and (37) together define a class of estimators $\hat{\mathcal{S}}_{\epsilon_{N}}$ parameterized by $\epsilon_{N}$. The solution of the optimization problem stated in $(38)$ is a minimax estimator in the restricted class $\hat{\mathcal{S}}_{\epsilon_{N}}$ of estimators.

Figure 3 reports the optimal values of $\epsilon_{N}$ as a function of $N$, with $\hat{\rho}_{0}$ defined as in (31) and (32) and restricted to the qubit case (labeled in Fig. 33 as 'Optimized $\epsilon_{N}$ for our estimator'). The performance of the estimator with the optimal value of $\epsilon_{N}$ is 


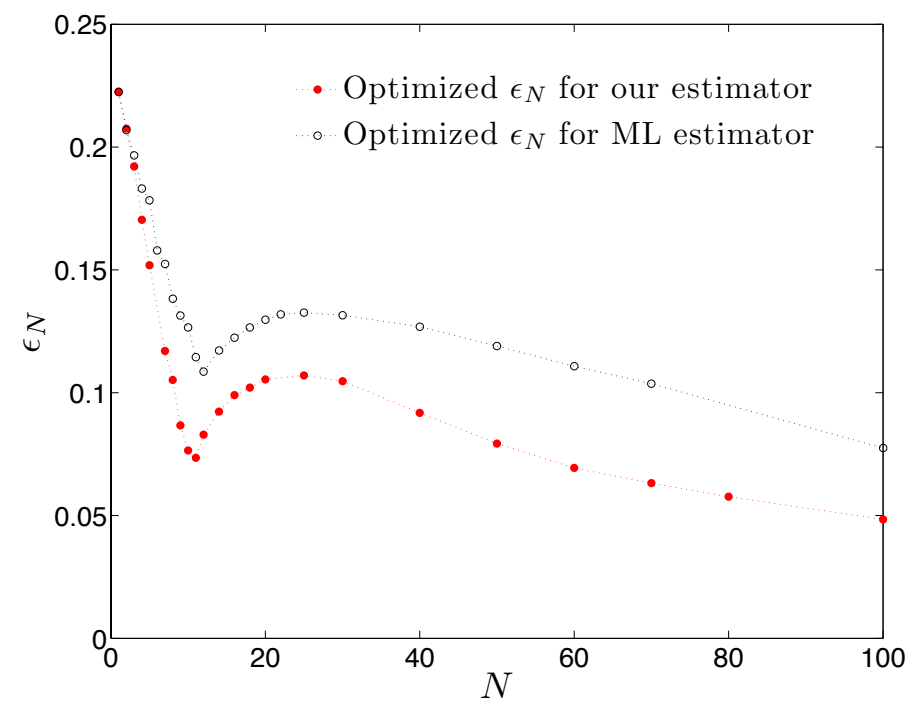

Fig. 3. Optimized value of $\epsilon_{N}$ for the qubit problem for different values of $N$, with $\hat{\rho}_{0}$ defined as in 31 and 32, as well as for the ML estimator modified by an $\epsilon_{N}$ parameter.

plotted in Fig. 2 (labeled in Fig. 2 as 'min, max risk'). Observe that as $N$ grows, the difference in performance between optimizing the value of $\epsilon_{N}$ and choosing $\epsilon_{N}=0$ (that is, the estimator discussed in Section 3.3 rapidly diminishes. If desired, for practical convenience, one can set $\epsilon_{N}=0$ for $N \gtrsim 100$. That $\epsilon_{N}$ approaches 0 as $N$ increases is particularly rewarding because it is in line with our intuition that for small $N$, we have little evidence that can support reporting a point estimator that is close to a rank-deficient state; however, as $N$ increases, we gather more and more data and gain confidence in reporting a state that is closer and closer to a rank-deficient state, as described by our estimator with $\epsilon_{N}$ approaching zero.

For comparison, we have also plotted the performance of the ML estimator, with the modification that one restricts the domain of the maximization of the likelihood function to states such that $p^{2} \leq \frac{1}{3}\left(1-\epsilon_{N}\right)$, where $\epsilon_{N}$ is again chosen via the same minimax procedure as above. This simple modification removes the problem of rank-deficiency of the usual ML estimator. In fact, as can be seen from Fig. 2 (line labeled 'min, max risk (ML with $\left.\epsilon_{N}\right)$ '), it significantly improves the maximum risk for the ML estimator, although it does not do nearly as well as the estimator discussed above.

Our approach to a restricted minimax estimator can be extended beyond the qubit case and beyond using a $\rho_{0}$ that comes from the classical minimax estimator. As mentioned in Section 3.3. one can begin with one's favorite classical estimator and admix enough of the completely mixed state to ensure physicality of the resulting estimator. To fix the rank-deficiency problem, one can then use a similar 
minimax procedure as in (38) to find the best estimator that avoids the physicality boundary. In the qubit case, a single parameter $\epsilon_{N}$ was sufficient to delineate the physicality boundary and characterize the relevant class of estimators. For higher dimension, physicality constraints are more complicated (and, in fact, are often not well understood), and one would typically require more than one parameter to define the analog of $\hat{\mathcal{S}}_{\epsilon_{N}}$. Nevertheless, the same minimax procedure is applicable.

Variants of our estimator are also possible. For example, one can treat both $\epsilon_{N}$ as well as $b_{N}$ (with $\left.a_{N}=1-b_{N}\right)$ in (31) as parameters that we choose in a minimax fashion. Another variant, for the case of the tetrahedron POM for the qubit, suggests itself when we examine (37) which determines $\lambda$ whenever $\hat{p}_{0}^{2}>\frac{1}{3}\left(1-\epsilon_{N}\right)$ : The choice $b_{N}^{2}=1-4 \epsilon_{N}$ gives a particularly simple value for $\lambda$ that depends only on the relative frequencies $\nu_{k}$, but not on the parameters $b_{N}$ and $\epsilon_{N}$. One then performs minimax optimization over $\epsilon_{N}$ only. Both variants give results very similar to our estimator above for the case of the tetrahedron POM for the qubit.

\section{Conclusion}

We demonstrated a simple procedure for adapting the minimax estimator for the classical die problem to the quantum case of a single qubit with the tetrahedron measurement. We obtained an estimator that inherited desirable properties from the classical version: (i) It is always full rank and contains a reasonable $N$ dependence; (ii) it has much smaller maximum risk, as measured by the mean squared error, compared to the popular ML estimator; (iii) it gives nearly constant risk over all states and hence treats all possible states in a fair manner.

The procedure of admixing a sufficient amount of the completely mixed state to obtain a physical and full-rank estimator can be applied to any estimator appropriate for the analogous classical problem. For typical data and most states, the classical estimator is usually physical; it is only the rare case that requires a physicality correction. This automatically ensures that the resulting quantum estimator will inherit most of the properties of the classical estimator. One can, for example, do this for estimators for the classical die problem that are minimax for other risk functions (for example, based on relative entropy). The procedure is also applicable beyond the qubit case and also beyond a SIC-POM. For higher dimensions, the physicality constraints will involve more inequalities that the probabilities $\left\{p_{k}\right\}$ must satisfy, but can, in principle, be imposed as additional constraints for the choice of the admixing parameter $\lambda$. In every case, a minimax procedure can be used to choose parameters like $\epsilon_{N}$ to avoid the boundary. Note also that, for problems with an unusual symmetry, one can in fact consider admixing not the completely mixed state but some other suitable reference state.

Given the simplicity of this estimator, we believe it will find much utility in tomographic experiments as a first-cut point estimate of the unknown state. Future work exploring the effectiveness of this procedure for other estimators, risk functions, and higher dimensions can also be potentially interesting. Progress towards 
general minimax estimators following the programme set up in this paper will also certainly be of importance to quantum tomography.

\section{Acknowledgments}

We are grateful for insightful discussions with David Nott and Benjamin Phuah. This work is supported by the National Research Foundation and the Ministry of Education, Singapore.

\section{Appendix A. Geometry of S-POMs}

The $K$ outcomes $\Pi_{k}$ of an S-POM for a $d$-dimensional system obey

$$
\begin{aligned}
\operatorname{tr}\left\{\Pi_{j} \Pi_{k}\right\} & =\frac{d}{K}\left[w \delta_{j k}+\frac{1-w}{K-1}\left(1-\delta_{j k}\right)\right], \\
\operatorname{tr}\left\{\Pi_{k}\right\} & =\frac{d}{K},
\end{aligned}
$$

with

$$
\frac{1}{K} \leq w \leq 1
$$

The lower bound applies when the $\Pi_{k}$ s are multiples of the identity, which is a case of no interest; the upper bound applies when the outcomes have support in pairwise orthogonal subspaces. If the outcomes are (subnormalized) rank- $r$ projectors, we have $w=d /(r K)$ with $1 \leq d / r \leq K$. Of particular importance is the rank-1 situation, for which

$$
\Pi_{k}^{2}=\frac{d}{K} \Pi_{k}, \quad \operatorname{tr}\left\{\Pi_{j} \Pi_{k}\right\}=\frac{d^{2}}{K^{2}}\left[\delta_{j k}+\frac{K-d}{(K-1) d}\left(1-\delta_{j k}\right)\right]
$$

hold.

The set of traceless hermitian operators constitute a real $\left(d^{2}-1\right)$-dimensionless vector space that we endow with the Hilbert-Schmidt inner product

$$
A \cdot B \equiv \operatorname{tr}\{A B\} \quad \text { for } A^{\dagger}=A, B^{\dagger}=B, \operatorname{tr}\{A\}=\operatorname{tr}\{B\}=0 .
$$

Since the operators $\Pi_{k}-1 / K$ are in this vector space, we can state A.1] as

$$
\left(\Pi_{j}-\frac{1}{K}\right) \cdot\left(\Pi_{k}-\frac{1}{K}\right)=\frac{d}{K} \frac{w K-1}{K}\left[\delta_{j k}-\frac{1}{K-1}\left(1-\delta_{j k}\right)\right] .
$$

In conjunction with $\sum_{k}\left(\Pi_{k}-1 / K\right)=0$, this tells us that the vectors $\Pi_{k}-1 / K$ define a flat $K$-edged pyramid, if we employ the terminology of Ref. 14. In the rank-1 situation of A.3, the prefactor in A.5 is $(d-1) d / K^{2}$.

In view of this geometrical property of the S-POM, there can be at most $d^{2}$ outcomes. Indeed, the S-POM is IC for $K=d^{2}$, but not when $K<d^{2}$, and there 
are no S-POMs with $K>d^{2}$. In an alternative way of reasoning, we represent the vectors $\Pi_{k}-\frac{1}{K}$ by the columns of the $K \times K$ matrix

$$
\sqrt{\frac{(w K-1) d}{(K-1) K^{3}}}\left[\begin{array}{cccc}
K-1 & -1 & \cdots & -1 \\
-1 & K-1 & \cdots & -1 \\
\vdots & \vdots & \ddots & \vdots \\
-1 & -1 & \cdots & K-1
\end{array}\right]
$$

and note that this matrix has rank $K-1$, which implies that the $K$ vectors $\Pi_{k}-\frac{1}{K}$ span a $(K-1)$-dimensional subspace.

Regarding the statistical operator $\rho$, we note that $\rho-1 / d$ is hermitian and traceless, and so are the operators $\Lambda_{k}-1 / d$ that appear in (4),

$$
\rho=\sum_{k=1}^{K} p_{k} \Lambda_{k}=\frac{1}{d}+\sum_{k=1}^{K}\left(p_{k}-\frac{1}{K}\right)\left(\Lambda_{k}-\frac{1}{d}\right),
$$

where either $p_{k}-1 / K \rightarrow p_{k}$ or $\Lambda_{k}-1 / d \rightarrow \Lambda_{k}$ is a permissible replacement, but not both. The defining property of the $\Lambda_{k} \mathrm{~s}$, namely $\operatorname{tr}\left\{\Pi_{j} \Lambda_{k}\right\}=\delta_{j k}$ or

$$
\left(\Pi_{j}-\frac{1}{K}\right) \cdot\left(\Lambda_{k}-\frac{1}{d}\right)=\frac{K-1}{K}\left[\delta_{j k}-\frac{1}{K-1}\left(1-\delta_{j k}\right)\right]
$$

implies their standard form,

$$
\begin{aligned}
\Lambda_{k} & =\frac{1}{d}+\frac{(K-1) K}{(w K-1) d}\left(\Pi_{k}-\frac{1}{K}\right) \\
& =\frac{(K-1) K}{(w K-1) d} \Pi_{k}-\frac{(1-w) K}{(w K-1) d} .
\end{aligned}
$$

If the S-POM is not IC $\left(K<d^{2}\right)$, the $\Lambda_{k}$ s are not uniquely determined, because there is then the option to add a traceless hermitian operator on the right-hand side of A.9 that is orthogonal to all $K$ vectors $\Pi_{k}-1 / K$. It follows that the statistical operator $\rho$ of A.7) is not unique unless the S-POM is a SIC-POM. What is unique, however, is the part of $\rho-1 / d$ that resides in the $(K-1)$-dimensional subspace spanned by the vectors $\Pi_{k}-1 / K$.

For the standard $\Lambda_{k} \mathrm{~s}$ of $\mathrm{A.9}$, the vectors $\Lambda_{k}-1 / d$ make up the same flat pyramid as the vectors $\Pi_{k}-1 / K$, except that the edges have different lengths. More specifically, we have

$$
\left(\Lambda_{j}-\frac{1}{d}\right) \cdot\left(\Lambda_{k}-\frac{1}{d}\right)=\frac{(K-1)^{2}}{(w K-1) d}\left[\delta_{j k}-\frac{1}{K-1}\left(1-\delta_{j k}\right)\right],
$$

and

$$
\frac{K}{\sqrt{(w K-1) d}}\left(\Pi_{k}-\frac{1}{K}\right)=\frac{\sqrt{(w K-1) d}}{K-1}\left(\Lambda_{k}-\frac{1}{d}\right)
$$

are the edge vectors of the generic pyramid with unit-length edges. 
In the qubit case $(d=2)$, the $\left(d^{2}-1\right)$-dimensional real vector space of traceless hermitian operators is isomorphic to the three-dimensional cartesian space in which the Bloch ball is embedded. Rank-1 outcomes are of the form

$$
\Pi_{k}=\frac{1}{K}\left(1+\vec{e}_{k} \cdot \vec{\sigma}\right)
$$

with $K=2$ for the von Neumann measurement, $K=3$ for the so-called trine measurement, and $K=4$ for the tetrahedron measurement of (25). The $\vec{e}_{k}$ s are unit vectors, with

$$
\left(\Pi_{j}-\frac{1}{K}\right) \cdot\left(\Pi_{k}-\frac{1}{K}\right)=\frac{2}{K^{2}} \vec{e}_{j} \cdot \vec{e}_{k}
$$

stating how the inner product in the operator space is related to the scalar product of three-dimensional vectors. When representing the $\vec{e}_{k}$ s by three-component columns of cartesian coordinates, possible choices are

$$
\begin{aligned}
& {\left[\begin{array}{ll}
\vec{e}_{1} & \vec{e}_{2}
\end{array}\right]=\left[\begin{array}{rr}
0 & 0 \\
0 & 0 \\
1 & -1
\end{array}\right] \quad \text { for } K=2, \quad\left[\vec{e}_{1} \vec{e}_{2} \vec{e}_{3}\right]=\frac{1}{\sqrt{6}}\left[\begin{array}{rrr}
2 & -1 & -1 \\
-1 & 2 & -1 \\
-1 & -1 & 2
\end{array}\right] \quad \text { for } K=3} \\
& \text { and }\left[\begin{array}{llll}
\vec{e}_{1} & \vec{e}_{2} & \vec{e}_{3} & \vec{e}_{4}
\end{array}\right]=\frac{1}{\sqrt{3}}\left[\begin{array}{rrrr}
1 & -1 & -1 & 1 \\
-1 & 1 & -1 & 1 \\
-1 & -1 & 1 & 1
\end{array}\right] \quad \text { for } K=4
\end{aligned}
$$

In each case, one easily confirms that $\sum_{k} \vec{e}_{k}=0$ and

$$
\vec{e}_{j} \cdot \vec{e}_{k}=\delta_{j k}-\frac{1}{K-1}\left(1-\delta_{j k}\right),
$$

as implied by A.13 with A.5.

\section{Appendix B. Proof of Fact 1}

Fact 1. Suppose we choose the square of the Euclidean distance to define the estimation error:

$$
E\left(\hat{\rho}, \rho, D_{N}\right)=\operatorname{dist}\left(\hat{\rho}\left(D_{N}\right), \rho\right) \equiv \operatorname{tr}\left\{\left[\hat{\rho}\left(D_{N}\right)-\rho\right]^{2}\right\} .
$$

Then, the unique Bayes estimator for prior distribution $\mathrm{d} \mu$ is the mean estimator $\hat{\rho}_{\mathrm{ME}}[\mathrm{d} \mu]$.

Proof. Consider any estimator $\hat{\rho} \in \hat{\mathcal{S}}$. Inserting $0=-\hat{\rho}_{\mathrm{ME}}[\mathrm{d} \mu]+\hat{\rho}_{\mathrm{ME}}[\mathrm{d} \mu]$ into the squared Euclidean distance, the estimation error can be written as

$$
\begin{aligned}
E\left(\hat{\rho}, \rho, D_{N}\right)=\operatorname{tr}\left\{\left[\rho-\hat{\rho}_{\mathrm{ME}}\left(D_{N}\right)\right]^{2}+\left[\hat{\rho}_{\mathrm{ME}}\left(D_{N}\right)-\hat{\rho}\left(D_{N}\right)\right]^{2}\right. \\
\left.-2\left[\rho-\hat{\rho}_{\mathrm{ME}}\left(D_{N}\right)\right]\left[\hat{\rho}_{\mathrm{ME}}\left(D_{N}\right)-\hat{\rho}\left(D_{N}\right)\right]\right\}
\end{aligned}
$$


The average risk can then be computed as

$$
\begin{aligned}
F_{N}(\hat{\rho}, \mathrm{d} \mu)= & \int \mathrm{d} \mu(\rho) \sum_{D_{N} \in \mathcal{D}_{N}} \mathcal{L}\left(D_{N} \mid \rho\right) \operatorname{tr}\left\{\left[\rho-\hat{\rho}_{\mathrm{ME}}\left(D_{N}\right)\right]^{2}\right\} \\
& +\int \mathrm{d} \mu(\rho) \sum_{D_{N} \in \mathcal{D}_{N}} \mathcal{L}\left(D_{N} \mid \rho\right) \operatorname{tr}\left\{\left[\hat{\rho}_{\mathrm{ME}}\left(D_{N}\right)-\hat{\rho}\left(D_{N}\right)\right]^{2}\right\} \\
& -2 \int \mathrm{d} \mu(\rho) \sum_{D_{N} \in \mathcal{D}_{N}} \mathcal{L}\left(D_{N} \mid \rho\right) \operatorname{tr}\left\{\left[\rho-\hat{\rho}_{\mathrm{ME}}\left(D_{N}\right)\right]\left[\hat{\rho}_{\mathrm{ME}}\left(D_{N}\right)-\hat{\rho}\left(D_{N}\right)\right]\right\} .
\end{aligned}
$$

The third term is zero, by definition of $\hat{\rho}_{\mathrm{ME}}[\mathrm{d} \mu]$. The first term does not depend on $\hat{\rho}$ and just gives a fixed constant value. To find the Bayes estimator, we thus solve the optimization problem

$$
\min _{\hat{\rho} \in \hat{\mathcal{S}}} \int \mathrm{d} \mu(\rho) \sum_{D_{N} \in \mathcal{D}_{N}} \mathcal{L}\left(D_{N} \mid \rho\right) \operatorname{tr}\left\{\left[\hat{\rho}_{\mathrm{ME}}\left(D_{N}\right)-\hat{\rho}\left(D_{N}\right)\right]^{2}\right\},
$$

for which $\hat{\rho}=\hat{\rho}_{\mathrm{ME}}[\mathrm{d} \mu]$ is clearly the unique solution.

\section{Appendix C. Proof of Fact 2}

Fact 2. An estimator with constant risk that is also a Bayes estimator for some prior distribution $\mathrm{d} \mu$ is a minimax estimator. If the estimator is also the unique Bayes estimator for some $\mathrm{d} \mu$, then it is the unique minimax estimator.

Proof. Suppose $\hat{\rho}_{\mathrm{B}}$ is a Bayes estimator for $\mathrm{d} \mu$ with constant risk, that is, $R_{N}\left(\rho, \hat{\rho}_{\mathrm{B}}\right)=R_{N, \mathrm{~B}}$ for all $\rho$ in $\mathcal{S}$. $\hat{\rho}_{\mathrm{B}}$ satisfies

$$
\int \mathrm{d} \mu(\rho) R_{N}\left(\rho, \hat{\rho}_{\mathrm{B}}\right)=R_{N, \mathrm{~B}}=\max _{\rho \in \mathcal{S}} R_{N}\left(\rho, \hat{\rho}_{\mathrm{B}}\right) .
$$

Consider another estimator $\hat{\rho} \neq \hat{\rho}_{\mathrm{B}}$. Then, we have that

$$
\begin{aligned}
\max _{\rho \in \mathcal{S}} R_{N}(\rho, \hat{\rho}) & \geq \int \mathrm{d} \mu(\rho) R_{N}(\rho, \hat{\rho}) \\
& \geq \int \mathrm{d} \mu(\rho) R_{N}\left(\rho, \hat{\rho}_{\mathrm{B}}\right)=\max _{\rho \in \mathcal{S}} R_{N}\left(\rho, \hat{\rho}_{\mathrm{B}}\right) .
\end{aligned}
$$

The first inequality is simply a statement that the maximum is greater then the mean; the second inequality follows from the fact that $\hat{\rho}_{\mathrm{B}}$ is a Bayes estimator. Equation C.2 says precisely that $\hat{\rho}_{\mathrm{B}}$ is minimax. If $\hat{\rho}_{\mathrm{B}}$ is also the unique Bayes estimator for $\mathrm{d} \mu$, the second inequality is converted into a strict inequality (">"), and we have $\max _{\rho \in \mathcal{S}} R_{N}(\rho, \hat{\rho})>\max _{\rho \in \mathcal{S}} R_{N}\left(\rho, \hat{\rho}_{\mathrm{B}}\right)$, which proves the uniqueness of $\hat{\rho}_{\mathrm{B}}$ as a minimax estimator.

\section{References}

1. M. Christandl and R. Renner, Reliable quantum state tomography, e-print arXiv:1108.5329[quant-ph] (2011). 
2. R. Blume-Kohout, Robust error bars for quantum tomography, e-print arXiv:1202.5270[quant-ph] (2012).

3. Z. Hradil, Quantum-state estimation, Phys. Rev. A 55 (1997) R1561.

4. Z. Hradil, J. Řeháček, J. Fiurášek, and M. Ježek, Maximum-Likelihood Methods in Quantum Mechanics, Lect. Notes Phys. 649 (2004) 59.

5. R. D. Cousins, Why isn't every physicist a Bayesian?, Am. J. Phys. 63 (1995) 398.

6. R. E. Kass and L. Wasserman, The selection of prior distributions by formal rules, J. Am. Stat. Assoc. 91 (1996) 1343.

7. E. T. Jaynes, Probability Theory: The Logic of Science, Cambridge University Press (2003).

8. G. M. D'Ariano, M. F. Sacchi, and J. Kahn, Minimax quantum state discrimination, Phys. Rev. A $\mathbf{7 2}$ (2005) 032310.

9. G. M. D'Ariano, M. F. Sacchi, and J. Kahn, Minimax discrimination of two Pauli channels, Phys. Rev. A 72 (2005) 052302.

10. M. Guţă and L. Artiles, Minimax estimation of the Wigner function in quantum homodyne tomography with ideal detectors, Math. Meth. Stat. 16 (2007) 1.

11. E. L. Lehmann and G. Casella, Theory of Point Estimation, Springer, 2nd edition (1998).

12. J. M. Renes, R. Blume-Kohout, A. J. Scott, and C. M. Caves, Symmetric informationally complete quantum measurements, J. Math. Phys. 45 (2004) 2171.

13. J. Řeháček, B.-G. Englert, and D. Kaszlikowski, Minimal qubit tomography, Phys. Rev. A 70 (2004) 052321.

14. B.-G. Englert and J. Řeháček, How well can you know the edge of a quantum pyramid?, J. Mod. Opt. 57 (2010) 218. 